\title{
TERRESTRIAL LASER SCANNING AND SATELLITE DATA IN CULTURAL HERITAGE BUILDING DOCUMENTATION
}

\author{
Aikaterini Karagianni \\ Aristotle University of Thessaloniki, Faculty of Engineering, School of Civil Engineering, \\ Lab. of Photogrammetry - Remote Sensing, Thessaloniki, Greece - aikateck@civil.auth.gr
}

KEY WORDS: Remote Sensing, Terrestrial Laser Scanning, Satellite Imagery, Building Documentation, Cultural Heritage.

\begin{abstract}
:
Technological advances in the field of information acquisition have led to the development of various techniques regarding building documentation. Among the proposed methods, acquisition of data without being in direct physical contact with the features under investigation could provide valuable information especially in the case of buildings or areas presenting a high cultural value. Satellite or ground-based remote sensing techniques could contribute to the protection, conservation and restoration of cultural heritage buildings, as well as in the interpretation and monitoring of their surrounding area. The increasing interest in the generation of $3 \mathrm{D}$ facade models for documentation of the built environment has made laser scanning a valuable tool for 3D data collection. Through the generation of dense 3D point clouds, digitization of building facades could be achieved, offering data that could be used for further processing. Satellite imagery could also contribute to this direction, extending the monitoring possibilities of the buildings' surrounding area or even providing information regarding change detection in large-scale cultural landscapes. This paper presents the study of a mansion house built in the middle of the 18th century in northwestern Greece, using terrestrial laser scanning techniques for facade documentation, as well as satellite imagery for monitoring and interpretation purposes. The scanning process included multiple external scans of the main facade of the building which were registered using artificial targets in order to form a single colored 3D model. Further process resulted in a model that offers measurement possibilities valuable to future plans and designs for preservation and restoration. Digital processing of satellite imagery provided the extraction of additional enhanced data regarding the physiognomy of the surrounding area.
\end{abstract}

\section{INTRODUCTION}

Building documentation is a powerful tool that could contribute to a variety of engineering issues, including among others urban planning, identification and interpretation of buildings, monitoring and management of sites and cultural landscapes, as well as protection, conservation and restoration of cultural heritage (Yang et al., 2014; Haddad, 2011; Núñez Andrés et al., 2012).

Technological advances regarding information acquisition of buildings presenting a cultural value have led to the development of various techniques such as topographic, photogrammetric and ground-based remote sensing techniques or even their combination (Alshawabkeh, 2006; Tapete et al., 2013). Especially remote sensing techniques may contribute to monitoring and interpretation of the site, while a combination of other conventional techniques (topographic surveys and measurements, photogrammetric methodologies) could also be used in structural monitoring (Tournas et al., 2010).

Data collection for the documentation of the built environment in three dimensions (3D), as well as the increasing demands in the generation of facade models, have turned the research interest to laser scanning, which could be considered an efficient method regarding the digitization of building facades through the generation of dense 3D point clouds.

The speed of acquiring high-density data and the automated processing which offers high accuracy have led to the wider use of terrestrial laser scanners (TLS) in building documentation (Pfeifer and Briese, 2007; Sgrenzaroli, 2005). Satellite imagery could also provide complementary information, yet necessary in terms of monitoring possibilities of the surrounding area or change detection in cultural landscapes (Karagianni, 2019).

This paper presents the study of a mansion house of cultural significance, built in the middle of the 18th century in northwestern Greece, using terrestrial laser scanning techniques for facade documentation. Satellite images were also exploited for monitoring and interpretation purposes. The scanning process included multiple external scans of the main facade of the building which were registered using artificial targets in order to form a single colored 3D model. Further process resulted in a model which could offer measurement possibilities valuable to future plans and designs for preservation and restoration. Digital processing of the satellite imagery provided the extraction of additional enhanced data regarding the physiognomy of the surrounding area.

\section{STUDY AREA}

The study area is located in medium-high altitude $(630 \mathrm{~m})$ in the north western part of Greece, region of Western Macedonia and it concerns a preserved mansion in Kastoria city and its surroundings (Figure 1). The city is located on a peninsula at the western shore of Lake Orestiada and it has a long cultural history, which is depicted in the older parts of the city.

Apart from its high architectural and urban value, it also presents historical, geographical and archaeological value through its landscape diversity (Tsigaridas, 2012). Rich elements of Byzantine culture (Byzantine Justinian walls and medieval churches) as well as traditional mansions of the 17th and 18th centuries, unique for their architectural design are located in the city center (Doltso and Apozari traditional 
districts) (Moutsopoulos, 1989). The lake which surrounds the city is included in the Natura 2000 network, offering unique habitats for many endangered fauna and avifauna species (Ponce de León, 2015).
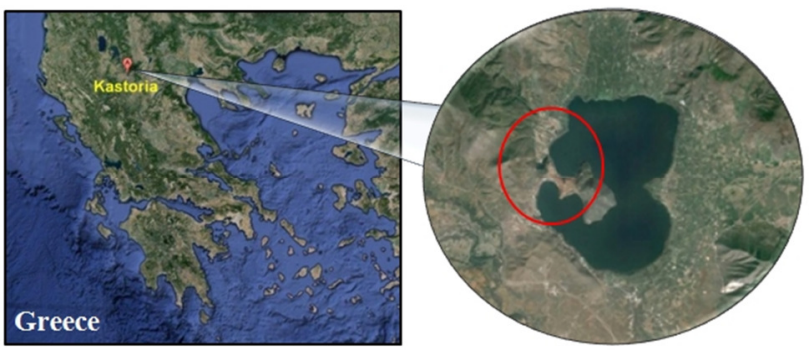

Figure 1. The location of the Regional Unit of Kastoria in Greece (left image) and the city of Kastoria in the red circle (right image) (Karagianni, 2019).

\subsection{Skoutaris Mansion Case Study}

Two main historic districts have been maintained in the city center of Kastoria, Doltso and Apozari, where mansions with local architecture preserve the traditional character of the area.

Skoutaris Mansion is located on the south side of the city, along the coast of the lake in the Doltso traditional district and it is a representative building of the area (Figure 2). The ground plan has a ' $\Pi$ ' shape, with windows on the first floor, while the windows on the second floor stand out for their larger size. The main entrance of the building is located on the south side, between the two basements. There is also a courtyard with vegetation both on the main side of the building and on the back.

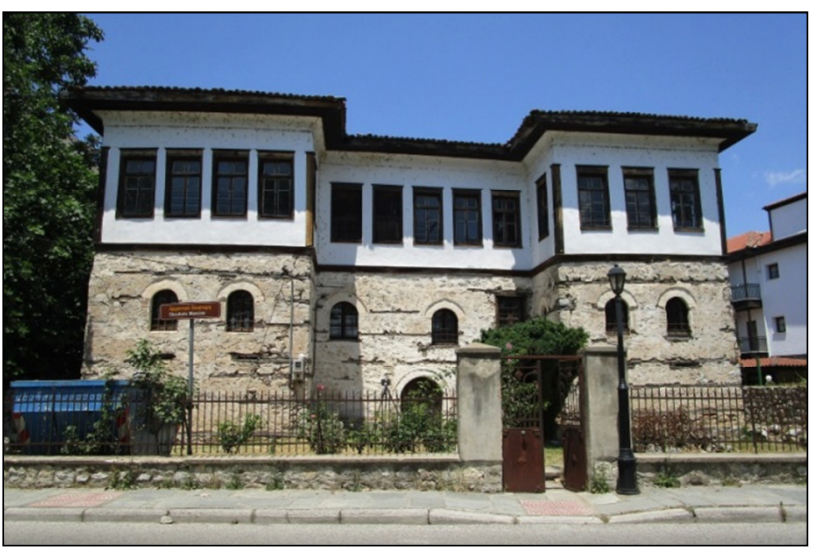

Figure 2. Skoutaris Mansion (image taken by the author).

\section{MATERIALS AND METHODS}

In order to extract information regarding the mansion, high resolution satellite data were used and terrestrial laser scanning techniques were exploited. The satellite images were used to locate the building, interpret the physiognomy of its surrounding area and monitor the district, while the terrestrial laser scanner was used to obtain 3D colored point clouds to give a realistic impression of the structure's facade and provide the ability to create highly detailed plans and designs.

\subsection{Satellite Data}

To get detailed information about the city of Kastoria and the area where the Skoutaris Mansion house is located, high resolution multispectral QuickBird satellite imagery was used that was acquired from DigitalGlobe on 2010-07-29 (Figures 3 and 4). It is a cloud-free, standard ortho-ready Level-2A product (radiometrically and geometrically corrected), with projection information: UTM, zone 34, spheroid \& datum WGS 84. QuickBird satellite was launched on October 18 of 2001. The sensor has 4 multispectral bands with a resolution of $2.44 \mathrm{~m}$ at nadir: Band 1 - Blue $(0.450-0.520 \mu \mathrm{m})$, Band 2 - Green $(0.520$ $0.600 \mu \mathrm{m})$, Band 3 - Red (0.630-0.690 $\mu \mathrm{m})$, Band 4 - Near Infrared (0.760-0.900 $\mu \mathrm{m})$ (QuickBird Information Sheet, 2021).

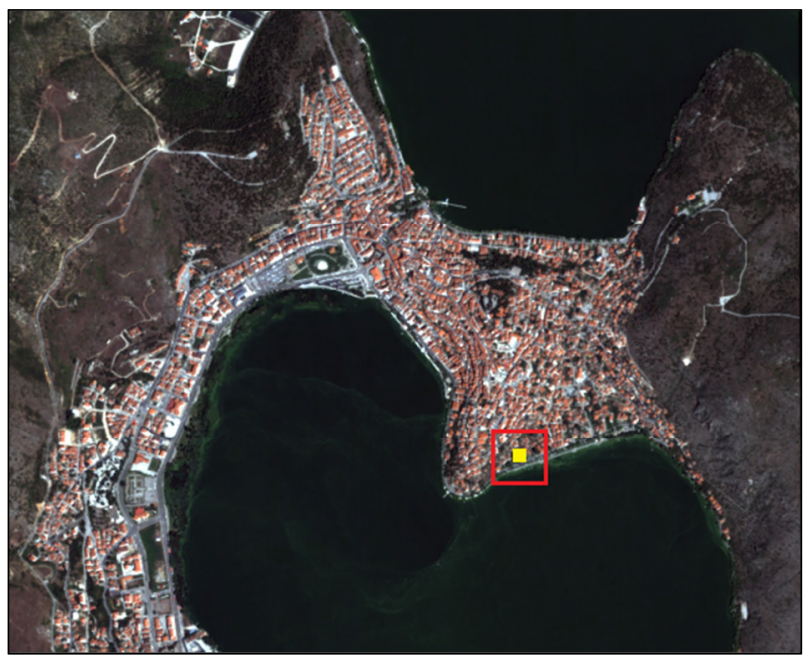

Figure 3. Position of Skoutaris Mansion (yellow dot) in the subset of QuickBird image covering the main part of the city of Kastoria.

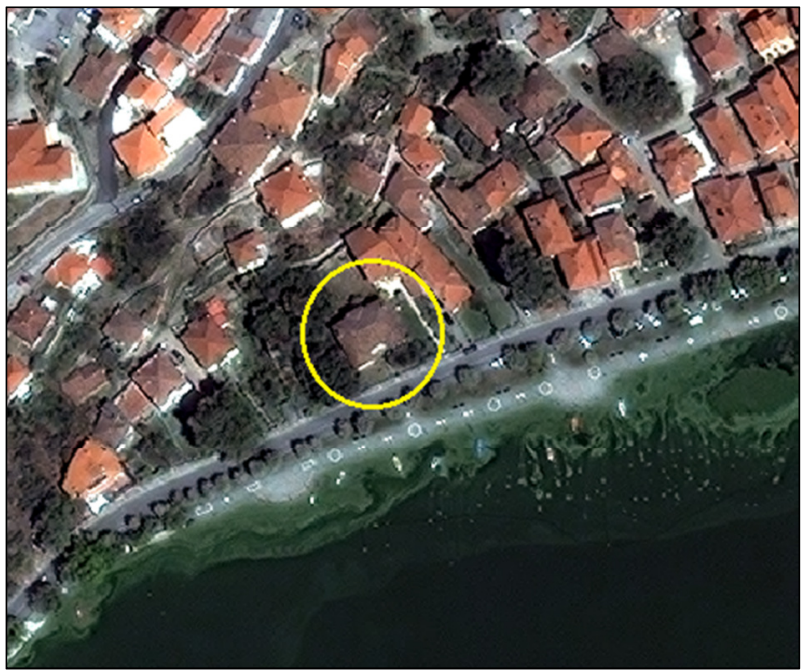

Figure 4. Skoutaris Mansion in the yellow circle (QuickBird subset image in true color composite).

The building is located on the south side of the city (traditional district of Doltso) near Orestiada Lake. The main entrance of the building is at the southeast and faces the main coastal road. The presence of vegetation around the mansion is evident. In addition, differentiation in the color of the roof of the building is noticeable, in comparison to the surrounding buildings. 
Further digital processing of the image was performed to extract useful information for additional interpretation and monitoring of the surrounding area. The original image was enhanced through decorrelation stretch algorithm, which applies a contrast stretch to the principal components of the image in order to decorrelate the bands and produce a strongly colored output image with high contrast using the Principal Components Transformation (ERDAS Field Guide, 2011). After the implementation of spectral enhancement, areas covered by vegetation are highlighted, buildings are delineated as shadows are minimized and color differentiations in the lake are more evident (Figure 5).

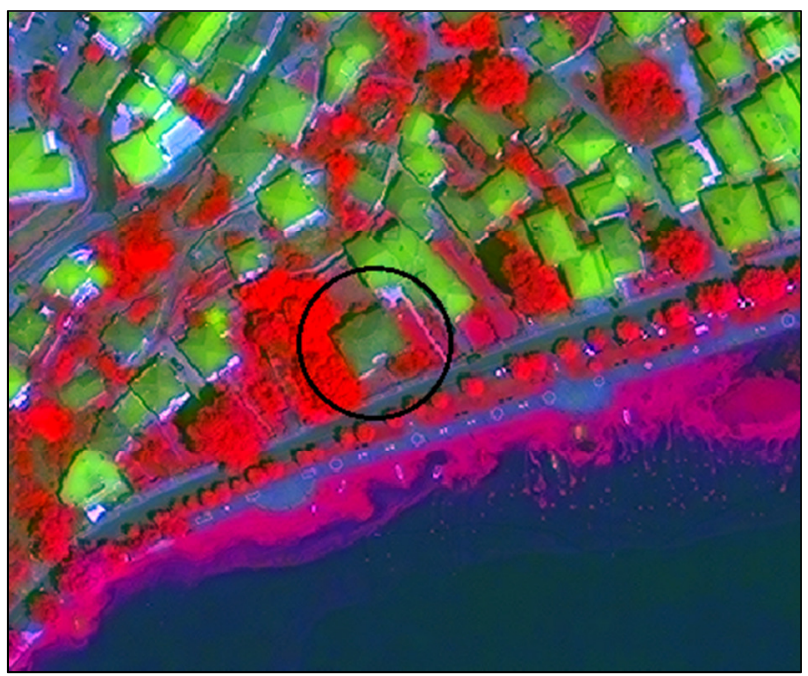

Figure 5. Skoutaris Mansion in the black circle (QuickBird subset image after decorrelation stretch).

The final step of digital processing included the construction of a model in order to extract information regarding the rooftops of the buildings sited in the surrounding area of Skoutaris Mansion. Through the exploitation of the high resolution that QuickBird imagery offers, an object-oriented approach was followed employing the spatial components in addition to spectral properties.

Based on the model, visual image interpretation cues for a feature are quantified, while machine learning components are trained using these cues which are ultimately applied to the imagery in order to extract the features. The quantification is achieved through algorithms which perform raster contouring, but also incorporate object and vector level processing in order to yield a spatially matched, precise shape depicting the studied features. The generated output accurately reflects the image content (IMAGINE Objective, 2010).

The workflow of this study included the following steps: raster pixel processing, segmentation, probability and size filtering, raster to vector conversion, vector object operations and vector clean-up operations. The results of the performed model are presented in Figure 6. The roof of Skoutaris Mansion in the yellow circle, as well as the roofs of the surrounding buildings, are depicted in red color.

The final layer representing the rooftops of the buildings could be very useful for interpretation purposes. Ambiguities related to the roof variations in size, color and shading due to different materials used or different orientations towards the sunlight were addressed using several representative roof samples.

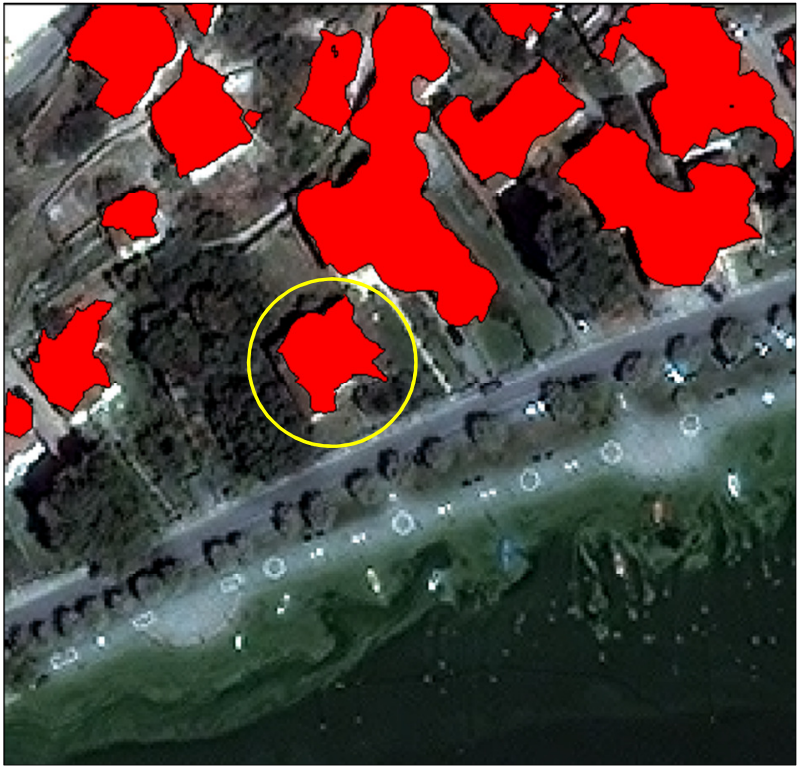

Figure 6. Roofs in red color acquired from QuickBird subset image through object-oriented feature extraction (roof of Skoutaris Mansion in the yellow circle).

The extracted information could extend the monitoring capabilities regarding the surrounding area and facilitate the detection of possible changes through time in future studies (time series analysis).

\subsection{Laser Scanner data}

Terrestrial laser scanning could be considered an effective tool in the civil engineering domain for digitizing large objects or entire scenes in order to acquire three-dimensional spatial information. The main steps that are followed include data acquisition, data process and data visualization (Staiger, 2003). TLS systems are active devices emitting their own radiation. They can accurately reconstruct scanned buildings through the generation of millions of 3D points, providing higher resolution and accuracy on building facades and low sloped roofs $(\mathrm{Pu}$, 2008). The 3D point clouds that are generated include points that have location coordinates in space, as well as color information and they represent the scanned building (Tournas et al., 2010).

In this study, FARO Focus 3D S120 phase-based laser scanner was used, which was provided by the Laboratory of Photogrammetry-Remote Sensing of the School of Civil Engineering in AUTH (Figure 7). The Focus 3D is a high-speed 3D laser scanner that uses advanced laser technology (class $3 R$ laser) for detailed measurement and documentation. It is an autonomous portable system able to acquire 3D point clouds of objects, producing an invisible laser beam with a wavelength of $905 \mathrm{~nm}$. Its range focus is between $0.60 \mathrm{~m}-120 \mathrm{~m}$, vertical field of view $305^{\circ}$ and horizontal $360^{\circ}$. It is characterized by a high measuring speed at a maximum of 976.000 measuring points per second and has a distance accuracy of up to $\pm 2 \mathrm{~mm}$.

The scanner is also equipped with an internal color camera giving the ability to produce photorealistic 3D color scans (color overlay). The generated point clouds and images are saved on the inserted SD memory card and thus can be easily transferred to another device (Faro Laser Scanner Focus3D User Manual, 2013). 


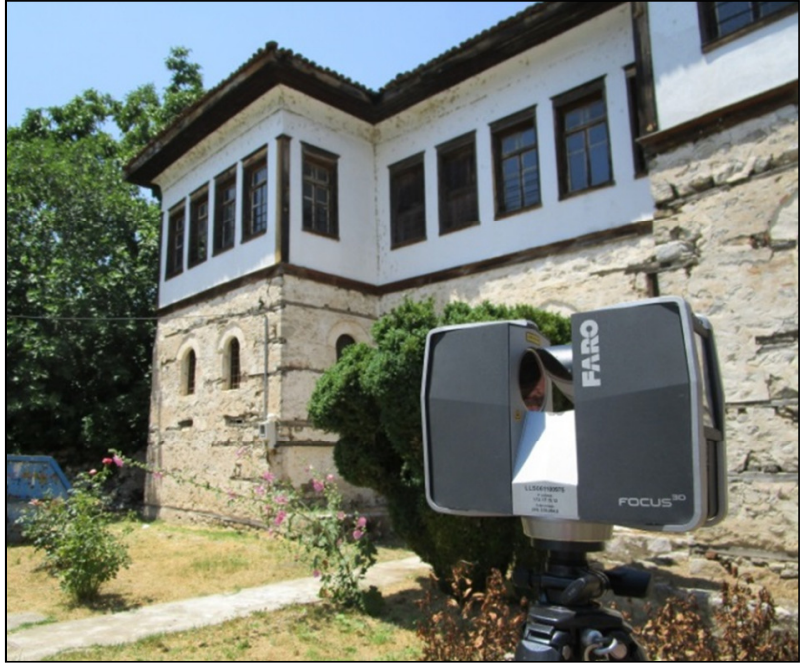

Figure 7. FARO Laser Scanner (image taken by the author).

The scanner was set up horizontally using a tripod. The scanning process included the inclination of laser pulses changed by a reflecting mirror, reflection of laser pulses on the surface of the building and reception of reflecting laser signals leading to an effect. Through this process, dense 3D coordinate information was acquired efficiently and accurately across the entire surface. Colored scan recording was also enabled, allowing the device to take color photos of the scanned environment with the integrated color camera. These photos were taken right after the laser scan and were used in the point cloud processing to automatically colorize the recorded scan data. The scan profile was set to outdoor conditions fitting the needs of the scene and the desired scan quality.

A total of four external scans were acquired to create the cloud of the main building facade. The position of the scanner in all four scans is presented in Figure 8. The scans were acquired consecutively and continuously in order to achieve the same external lighting conditions. The duration of each scan was approximately 8 minutes (expected scan time is in accordance with the selected resolution, quality value and scan range).

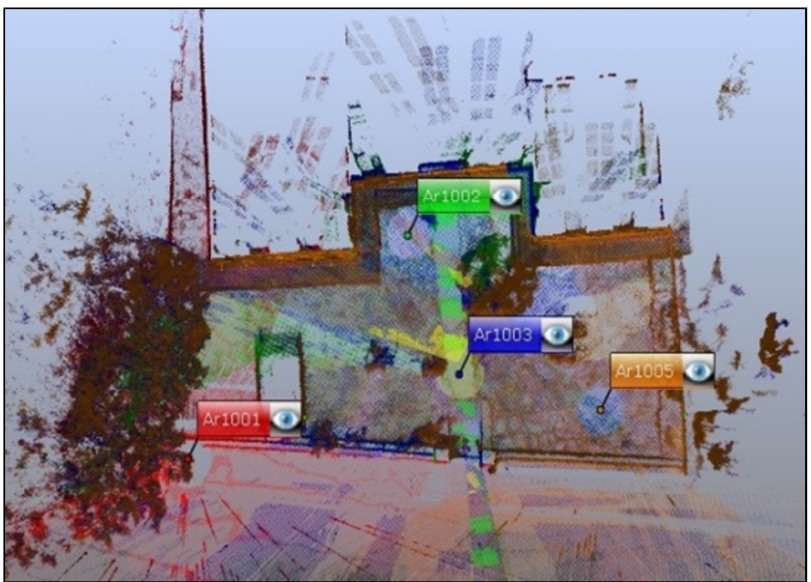

Figure 8. Scan locations in Skoutaris Mansion.
In order to ensure that the system would generate reference points and register signal contacts, artificial targets were placed in the scene. The targets that were used to extract distinct points were five spheres of bright material. The spheres were placed in positions that covered the scene horizontally and vertically, at different heights and were completely visible in all scans (Figure 9).

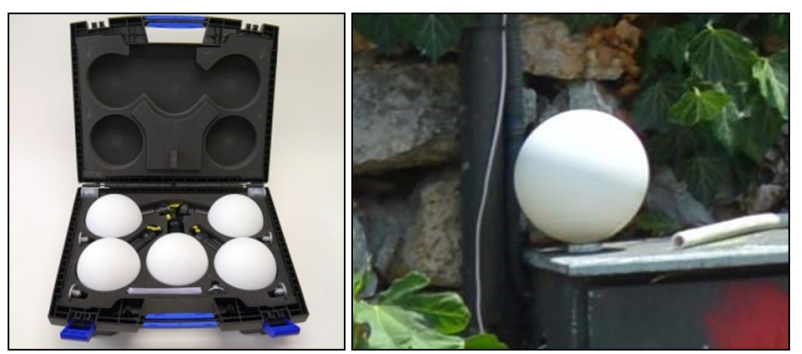

Figure 9. Reference spheres (images taken by the author).

\section{RESULTS}

The acquired data that carried highly detailed spatial information were imported into SCENE and Pointools Edit software programs for further processing which included point cloud visualization. A unified point cloud was obtained through the control points, the coordinates of which guided the registration of the four adjacent point clouds. The transformation of raw point clouds into a unified coordinate system uses scanned targets in a local coordinate system to solve the transformation parameters. After registering the scans using corresponding points, the software constructs a nonredundant surface representation, where each part of the measured object is only described once. The scanned building in $3 \mathrm{D}$ along with its surrounding landscape is presented in Figure 10 .

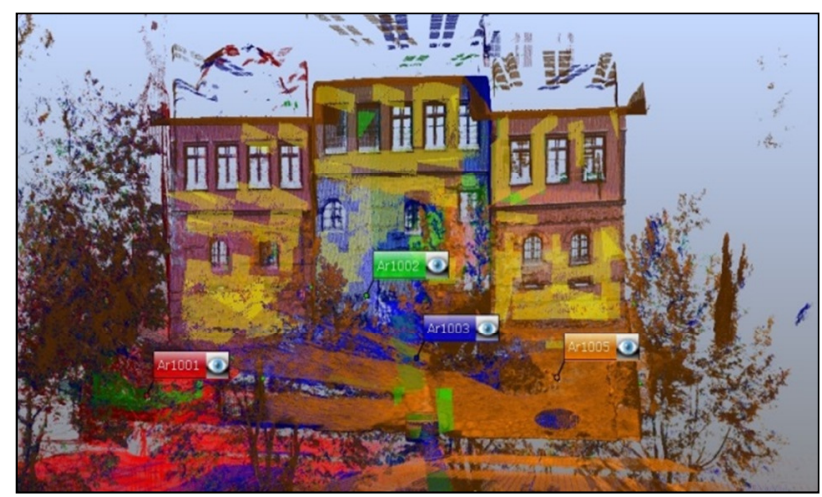

Figure 10. Scanned Skoutaris Mansion in 3D and surrounding landscape.

Table 1 presents the Scan Point Statistics and Table 2 includes the thresholds for point error and overlap, according to FARO SCENE 6.2 software manual (Faro SCENE User Manual, 2016). Point error values and overlap value in this study are within the accepted thresholds. 


\begin{tabular}{|c|c|}
\hline & Skoutaris Mansion \\
\hline Maximum Point Error & $6.1 \mathrm{~mm}$ \\
Mean Point Error & $4.8 \mathrm{~mm}$ \\
Minimum Overlap & $42.1 \%$ \\
\hline
\end{tabular}

Table 1. Scan Point Statistics.

\begin{tabular}{|c|c|c|}
\hline Point Error & $<8 \mathrm{~mm}$ & $>20 \mathrm{~mm}$ \\
\hline Overlap & $>25.0 \%$ & $<10.0 \%$ \\
\hline
\end{tabular}

Table 2. Thresholds for point error and overlap (FARO SCENE User Manual, 2016).

The main facade of Skoutaris Mansion after the scanning process is presented in Figure 11. The generated model can be used for measuring distances and angles by clicking onto distinct points. Employing facade's ortho-projection, measurements of basic elements can be done directly onto the image. The measurement capabilities of the building, in combination with the detection and monitoring capabilities offered by enhanced satellite images of the area could provide useful information for the restoration and preservation of the mansion in the future.

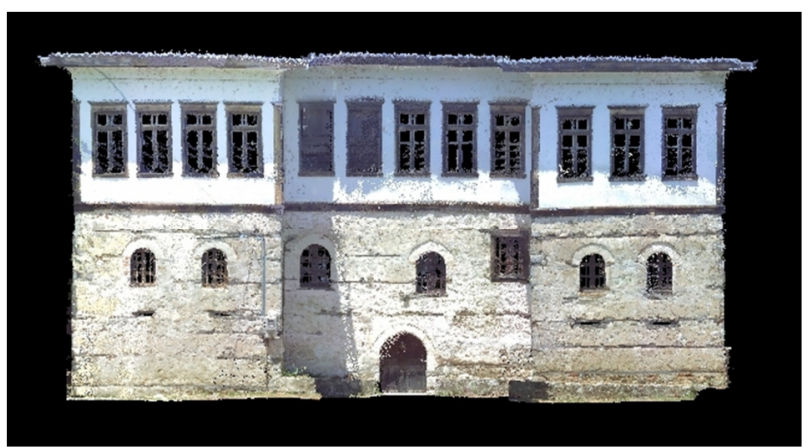

Figure 11. Skoutaris Mansion main facade.

\section{CONCLUSIONS}

During the last decade, TLS techniques have played a leading role in building documentation, offering valuable tools regarding civil engineering issues such as urban planning, monitoring, reconstruction, preservation and restoration of buildings.

Cultural heritage documentation in particular could benefit from the extraction of useful information through the exploitation of terrestrial laser scanning along with satellite data. New digital technologies can provide fast and accurate 3D documentation and measurement capabilities of space and structures compared to conventional survey techniques. TLS is a non-invasive technique that allows continuous monitoring without coming into physical contact with the scanned object (very useful especially in unreachable places).

Acquisition of detailed information for the comprehensive determination of structures' facades and types of materials can be achieved by generating reliable and detailed 3D models. Furthermore, the acquired data in digital format can be integrated into $\mathrm{CAD}$ systems for measurements or change detection studies (e.g. 3D models of cultural buildings acquired before and after the restoration).
This paper presents the study of a mansion house of cultural significance using TLS techniques for facade documentation and satellite data for complementary information of the surrounding area. Four 3D point clouds were generated with FARO Focus 3D laser covering the main facade of the building and were connected through the overlapping areas acquired from the different station points (point cloud registration).

In order to extract additional enhanced data regarding the building's environment and physiognomy of the surrounding area, satellite data of high spatial resolution were exploited. Further digital processing was applied to facilitate image interpretation. The spectral enhancement technique that was considered effective for this study was decorrelation stretch.

The exploitation of satellite imagery in order to locate the building and interpret its surrounding area could offer complementary, yet necessary information to the overall study. New satellites and sensors with various capabilities, high spatial resolution imagery, the policy of free data and other constantly evolving technologies in Earth Observation could provide continuous information, enabling the monitoring of changes in the surrounding area of the structures being studied. In addition, object-based image classification and feature extraction capabilities that are enabled through high spatial resolution imagery could build and maintain accurate geospatial content which could be directly merged into other geographic systems with minimal post-processing.

Especially in the case of cultural heritage buildings and the constraints related to the preservation of their physiognomy, the exploitation of satellite data can provide useful information on this subject through time series analysis and continuous remote monitoring. In addition, satellite data combined with TLS techniques can contribute to further studies regarding building extraction and 3D city modeling.

As the acquired point clouds contained information only about the geometry and the reflectivity of the surface, the caption of a series of images through the built-in digital camera followed the scanning process in order to get more information about the color of the surface and link it to the points. The final point cloud representing the main facade of the Skoutaris Mansion could be used in a variety of applications and offer effective measurement capabilities of the surface geometry. Furthermore, additional scans including the other facades of the building could be used for spatial and statistical analysis, Building Information Modeling (BIM) or could be integrated into Geographic Information Systems (GIS). The scanned structure can also be presented in a navigable 3D mode in order to facilitate the observation and interpretation of the building or generate a navigable model that could be used for virtual tourism purposes (virtual building visualization).

Restrictions and limitations that could appear in TLS techniques include among others difficulties in obtaining all the perimetrical facades of the building due to other buildings or obstacles, errors due to incorrect artificial target placement and poor registration (uneven distribution of the targets around the scene), errors that occur in the case of buildings with high or sloped roofs, as well as missing information for areas that could not be scanned from any station point.

In this study, in order to face the problems that may occur due to insufficient acquisition of data during the scanning process in the areas of the facade covered by the thick bush and the debris container in front of the structure, two additional scanning 
stations were used (Ar1002 and Ar1003). Increasing the number of scans facilitated the record of the points that could not be captured from the initial scanning locations, resulting in a complete representation of the main facade. Regarding difficulties in scanning procedure that could appear due to existing buildings or other obstacles in close proximity to the scanned building, a combination of traditional terrestrial techniques and structure-from-motion (SFM) photogrammetry could be used.

In conclusion, the exploitation of TLS techniques and digital processing of satellite imagery could contribute to the information collection and digital inventory technologies for the restoration and preservation of cultural heritage buildings.

\section{ACKNOWLEDGEMENTS}

The author wishes to thank the Ephorate of Antiquities of Kastoria for providing permission to acquire the necessary data and acknowledge the support of surveying engineer A. Sidiropoulos for his valuable help in data collection.

\section{REFERENCES}

Alshawabkeh, Y., 2006. Integration of Laser Scanning and Photogrammetry for Heritage Documentation. Doctoral Thesis, Institute of Photogrammetry, University of Stuttgart.

ERDAS Field Guide ${ }^{\mathrm{TM}}$, 2013. Intergraph Corporation, Erdas Inc. U.S.A, 445-446.

Faro Laser Scanner Focus3D User Manual, 2013. FARO Technologies Inc.

Faro SCENE User Manual, 2016. FARO Technologies Inc., 40.

Haddad, N.A., 2011. From ground surveying to 3D laser scanner: A review of techniques used for spatial documentation of historic sites. Journal of King Saud University-Engineering Sciences, 23(2), 109-118.

IMAGINE Objective User's Guide, 2010. Erdas Inc. U.S.A.

Karagianni, A., 2019. Research on the potentials of remote sensing techniques and interpretation of optical satellite data in civil engineering issues. Doctoral Thesis, Aristotle University of Thessaloniki.

Moutsopoulos, N., 1989. Kastoria: Greek Traditional Architecture. Melissa Publishing House, Athens.

Núñez Andrés, A., Buill Pozuelo, F., Regot Marimón, J., de Mesa Gisbert, A., 2012. Generation of virtual models of cultural heritage. Journal of Cultural Heritage, 13, 103-106.

Pfeifer, N. and Briese, C., 2007. Laser scanning-Principles and applications. Proc. 3rd International Exhibition and Scientific Congress GeoSiberia, Novosibirsk, Russia, 25-27 April, 2007, 93-112.

Ponce de León, P., 2015. Institutional framework and protection of traditional settlements-the case of Kastoria. Proc. Protection of Traditional Settlements and Contemporary Architectural Design, Society for the Environment and Cultural Heritage, Kastoria, Greece, 1-13.
$\mathrm{Pu}, \mathrm{S}$., 2008. Generating building outlines from terrestrial laser scanning. International Archives of Photogrammetry, Remote Sensing and Spatial Information Sciences, XXXVII (Part B5), Beijing, China, 3-11 July, 2008, 451-456.

QuickBird Information Sheet, 2021. DigitalGlobe Advanced Ortho Series. https://earth.esa.int/eogateway/documents/20142/ 37627/DigitalGlobe-Advanced-Ortho-Series.pdf (3 May 2021).

Sgrenzaroli, M., 2005. Cultural heritage 3D reconstruction using high resolution laser scanner: New frontiers data processing. Proc. CIPA 2005 XX International Symposium, Torino, Italy, 26 September-1 October, 2005, 1-6.

Staiger, R., 2003. Terrestrial laser scanning technology, systems and applications. Proc. 2nd FIG Regional Conference, Marrakech, Morocco, 2-5 December, 2003, 1-10.

Tapete, D., Casagli, N., Luzi, G., Fanti, R., Gigli, G. and Leva, D., 2013. Integrating radar and laser-based remote sensing techniques for monitoring structural deformation of archaeological monuments. Journal of Archaeological Science, 40(1), 176-189.

Tournas, E., Tsakiri, M. and Efessiou, I., 2010. The use of terrestrial laser scanning in the renovation of historic buildings. Proc. 8th International Symposium on the Conservation of Monuments in the Mediterranean Basin: Monument Damage Hazards \& Rehabilitation Technologies, Patras, Greece, 31 May-2 June, 2010, 1-13.

Tsigaridas, E., 2012. Kastoria through past and present times. ThessPrint S.A., Municipality of Kastoria.

Yang, H., Xu, X. and Neumann, I., 2014. The benefit of 3D laser scanning technology in the generation and calibration of FEM models for health assessment of concrete structures. Sensors, 14(11), 21889-21904. 